\section{ESCALA Y DISCONTINUIDAD ${ }^{1}$}

SCALE AND DISCONTINUITY

\section{JACQUES REVEL •}

Director de Estudios en la École des Hautes Études en Sciences Sociales (EHESS) de París (Francia).

Email: revel@ehess.fr

\section{Resumen}

A partir de la reflexión sobre los usos de las escalas de observación en el oficio historiográfico, desde aquellas propuestas por la microhistoria a los enfoques de la Historia Global, el texto interroga los modos en que desde diversos registros la narración historiográfica ha reconstruido diferentes procesos sociales - entre ellos el desarrollo del Estado moderno, del éxodo rural, de la industrialización y de la urbanización- recuperando las argumentaciones que ponen entre signos de interrogación el carácter necesario y universal de los mismos. Este ejercicio analítico permite mostrar cómo el rescate de las discontinuidades y desfasajes entre los diversos niveles de la materia histórica que estos hacen explícitos se constituye en un instrumento crítico frente a las evidencias simplificadoras de la realidad del mundo histórico.

\section{Registro bibliográfico}

REVEL, JACQUES «Escala y discontinuidad», en: ESTUDIOS SOCIALES, revista universitaria semestral, año XXX, $n^{\circ} 58$, Santa Fe, Argentina, Universidad Nacional del Litoral, enero-junio, 2020, pp. 141-153.

\section{Abstract}

From reflection on the uses of observation scales in the Historiography, as of those proposed by Microhistory to the approaches of Global History, the text questions the ways in which historiographical narration has reconstructed different social processes - for instancethe development of the modern State, the rural exodus, industrialization and urbanization- recovering thearguments that questions their necessary and universal character. This analytical exercise allows pointing out how the rescue of the discontinuities and mismatches between the different levels of historical matter constitutes a critical instrument against the simplifying evidence of the reality of the historical world.

\section{Descriptores · Describers}

Escalas / discontinuidad / historiografía Scales / discontinuity / Historiography

Recibido: 09/12/2019 Aprobado: 12 / 03 / 2020

1] Una versión preliminar de este trabajo fue publicada en inglés como «Multiple narratives: Scale and discontinuity in history», en: Sebastian Jobs, Alf Lüdtke (Eds.), Unsettling History, Archiving and narrating in historiography, Francfort/New York, Campus Verlag, 2010, p. 49-61. 
El debate acerca de las escalas de observación y de análisis del mundo social -y más específicamente, del mundo histórico- no es nuevo, incluso si conoció, desde hace una generación, una intensidad nueva y si fue objeto de reformulaciones significativas. Desde el final de los años 1970, el programa de la microhistoria había sido recibido como una propuesta nueva, incómoda, quizá simplemente porque rompía explícitamente con algunas convenciones, a veces tácitas, de las concepciones dominantes de la historia social. En el transcurso de los años I990, como en un juego pendular, es la perspectiva de una historia global la que parece regresar al primer plano, con una serie de propuestas cuyos títulos podían ser diferentes (World History, Global History, Connected Histories, historias cruzadas, etc.) y en los que expectativas metodológicas y programas no se superponían exactamente pero que tenían en común el hecho de reivindicar la consideración de fenómenos masivos, de vastos espacios, de duraciones largas.

Significativamente, el XIX Congreso internacional de Ciencias Históricas, que tuvo lugar en Oslo en el año 2000, eligió presentar como primer tema de reflexión propuesto a sus miembros: «Perspectives on Global History: Concepts and Methodology/Mundialización de la historia: conceptos y metodología». Por supuesto, sería demasiado simple — francamente hablando, simplificador - no ver ahí más que los efectos de una moda historiográfica, e incluso una serie de oscilaciones alrededor de lo que podría ser un punto de equilibrio ilusorio.

La tesis que quisiera defender aquí es que estas propuestas, que son las más exhibidas (y que son ciertamente percibidas) como alternativas, incluso antagonistas, reenvían todas juntas a un conjunto común de interrogaciones sobre la naturaleza y sobre el funcionamiento de los objetos sociales que elegimos estudiar y sobre los enfoques que intentan brindar respuestas a nuestras interrogaciones.

\section{II}

Este debate, ya lo dije, no es nuevo. Sin remontarnos tan lejos en el tiempo, recuerdo que muy pronto se cumplirán setenta años de la publicación de la tesis de Fernand Braudel, El Mediterráneo y el mundo mediterráneo en la época de Felipe II (1949) en su primera versión. Este libro fue recibido, y lo sigue siendo significativamente hoy, como un manifiesto para una historia que, sea cual fuere el marco 
geográfico y cronológico anunciado en su título, proponía no fijar otros límites más allá de los que requería la comprensión de su tema: el Mediterráneo de Braudel es susceptible, en función de las necesidades del análisis, tanto de dilatarse desde el África de las savanas hasta el Asia central y el continente americano de los primeros tiempos de la colonización; del mismo modo, algunos de los datos recogidos para la segunda mitad del siglo XVI se vuelven a situar en una duración más extensa. La obra de Braudel es sin duda una de las que más explícitamente puso en el centro de la reflexión de los historiadores la preocupación por aprehender las realidades históricas a través de unos marcos analíticos que las superan con creces (la «larga duración» o más tarde, la «economía-mundo»), y de los que se espera que tornen posible restituirles una más justa perspectiva.

El historiador mexicano Luis González y González es probablemente menos conocido que Fernand Braudel. Fue el autor de una de las primeras obras que se reivindicaron como "microhistoria». Pueblo en vilo. Microhistoria de San José de Gracia — curiosamente traducido al francés como Les barrières de la solitude. Histoire universelle de San José de Gracia, village mexicain (!) — fue publicada en 1968. Se trataba del estudio monográfico de una comunidad aldeana de Michoacán, en México central, seguida durante cuatro siglos y llevada a cabo con la convicción de que una observación directa sería capaz de restituir una parte ignorada u oculta de la existencia social: una parte que el autor no dudaba en caracterizar como matria, femenina, próxima, familiar, afectiva ${ }^{2}$. González no era, ciertamente el inventor del género de la monografía aldeana, de las que se sabe que está bien instalada en las costumbres historiográficas. Pero lo que me parece interesante en él, es la convicción que otra historia es posible a partir de la elección de un punto de vista particular. Nada nos obliga a seguirlo en su proyecto de recuperar en el marco de la comunidad de residencia el «elemento femenino, conservador, terrestre, dulce, obscuro y doloroso de la vida social». Lo que debemos considerar, por el contrario, es el vínculo establecido entre un marco de análisis y las realidades de las que él pretende dar cuenta.

Este doble recordatorio, entre muchos otros que serían posibles, no intenta sugerir que todo ha sido dicho, que la reflexión historiográfica no haría más que

2] Luis González y González desarrolló su concepción de la microhistoria en una serie de obras, entre las cuales pueden destacarse: Invitación a la microhistoria y Nueva invitación a la microhistoria (GONZÁLEZ Y GONZÁLEZ, 1973 y 1982). 
regresar periódicamente sobres sus propias huellas que habría olvido mientras tanto. Tampoco pretenden legitimar una suerte de decisión metodológica soberana tal como la elección de un partido que descalificaría desde el comienzo todas las demás opciones posibles. Cierto, Braudel no pensaba muy bien de una «microhistoria» que él se apresuraba a identificar con la "pequeńa-historia», o incluso todavía con la historia evenementielle de los historiadores tradicionales ${ }^{3}$. Sin duda, González tenía la intención de dotarse de los medios para escribir otra historia, que se situaría en contrapunto con los grandes relatos de la historia nacional, de las luchas políticas que comandaron y comandan todavía la construcción del pasado mexicano. Uno y otro tuvieron claramente consciencia de este hecho esencial tan especialmente recordado precedentemente por Arnoldo Momigliano: hacer la elección de una historia particular, es de hecho, eliminar —o es, por lo menos suspender, por hipótesis - una pluralidad de otras posibles historias. Pero es mejor que esas decisiones sean explícitas y razonadas. Después de todo, Braudel no dejó de argumentar las razones de su elección, de explicar los beneficios del conocimiento que él esperaba del acercamiento por la larga duración y los vastos espacios, de justificar los sacrificios que él había consentido. Se puede decir algo similar de González, y más todavía, de las microhistorias italianas a partir de mediados de los años de 1970, menos a través de los textos programáticos que produjeron y que al fin de cuentas permanecen poco numerosos, que del conjunto de argumentos que acompañaron sus elecciones y sus deslindes de los enfoques de los historiadores profesionales.

Desde el siglo XIX, los historiadores se dotaron de un cierto número de instrumentos analíticos, que estaban destinados a brindarles indicios sobre las realidades estudiadas. Esta tendencia se ha acentuado y acelerado en el siglo xx, en una confrontación ininterrumpida con las ciencias sociales. Estos instrumentos han sido y lo son actualmente, de naturaleza muy diversa: pueden ser unas herramientas técnicas de tratamiento de datos, de categorías descriptivas o analíticas, o incluso de modelos, es decir de conjuntos de hipótesis vinculadas entre sí. Su rol no es reproducir la realidad del pasado sino de procurarnos una versión inteligible, o, para retomar una feliz expresión de Bernard Lepetit, una «copia teórica» susceptible de ser luego sometida a una validación empírica. La historia cuantitativa o serial no conoce, sin duda, actualmente, la popularidad que tenía hace una generación. Pero

3] Al respecto: BRAUDEL (1972: 112 y passim). 
es importante reconocer que independientemente de los resultados factuales que produjo —o quizá más bien por encima de estos resultados— ella tuvo el efecto de transformar profundamente la relación que los historiadores mantenían con sus fuentes y con los objetos que se daban y se construían. Por supuesto, lo mismo puede decirse de las solicitudes hechas a las conceptualizaciones tomadas prestadas de la economía, de la sociología o de la antropología. Se puede decir también que las tentativas experimentales para descomponer el tiempo histórico a partir de la recuperación de distintos tipos de ciclos de la actividad económica indujeron las duraciones sociales diferenciales (fue el caso de la enseñanza de Francois Simiand y, luego de él, de Ernest Labrousse), o mencionar incluso, la gran construcción temporal propuesta por Fernand Braudel, desde El Mediterráneo a la trilogía que dedicó a la historia del capitalismo.

Después de estos ejemplos ya antiguos, los instrumentos se volvieron más sofisticados, los útiles se afinaron considerablemente, se trate del análisis de las series temporales, de las tentativas de modelización econométrica de la New economic History o incluso de los recursos a los modelos de parentesco elaborados por los antropólogos para dar cuenta de los mecanismos de las alianzas y la transmisión de bienes en las sociedades complejas. Los procedimientos de este tipo, de los que sería posible hoy multiplicar los ejemplos, tienen en común que se basan en un enfoque constructivista: es al historiador —o al antropólogo, o al sociólogo— que le corresponde seleccionar los datos que requiere la validación de su hipótesis de partida. Sobre este punto, probablemente, podemos estar todos de acuerdo sin demasiada dificultad, incluso si el programa de una historia cuantitativa pareció, a veces, olvidar en el camino estos requisitos elementales. Dichos procedimientos tienen en común introducir en la aprehensión del mundo sociohistórico una perspectiva discontinua que creo esencial. Reconstruir la historia del Mediterráneo, como lo hizo Braudel, según tres regímenes diferentes de duración, sin duda ligados entre ellos, pero diferenciados por las necesidades del análisis para dar cuenta de las temporalidades múltiples de la experiencia social, es exactamente introducir la discontinuidad y tomarla como un instrumento analítico, definiendo, por hipótesis, tres planos operatorios de los que luego es posible apreciar y comparar los beneficios heurísticos que cada uno de ellos puede brindar.

Ahora bien, me parece evidente que esta dimensión discontinua es más difícilmente aceptada por los historiadores que la dimensión constructivista de la que ella es, a mi juicio, sin embargo, inseparable. A esto se suma toda una serie de 
razones: el viejo espejismo de una «resurrección integral del pasado », incluso si ya no retomamos por nuestra cuenta la fórmula de Michelet conservando la idea de «hacerle la competencia al estado-civil», la convicción, mucho tiempo alcanzada por la seducción de los grandes modelos funcionalistas, de que era posible - y deseable - dar cuenta de una sociedad, de un momento histórico, de una situación, de una biografía, como de una totalidad; la idea de que entre las partes y el todo, existen unas relaciones casi orgánicas de homología, idea que está presente detrás de numerosas de las formas de ejemplificación, luego de generalizaciones a las que recurrimos permanentemente y en la mayoría de los casos sin reflexionar demasiado.

La tesis, bastante modesta, que yo quisiera defender aquí es que el principio de la variación de escalas, el juego razonado sobre las escalas de observación y del análisis de lo social, produce discontinuidad que es de donde obtiene su eficacia.

III

En un libro que dominó la producción de historia social en la segunda mitad del siglo xx, el historiador británico Edward P. Thompson estudió la formación de la clase obrera inglesa ${ }^{4}$. Sus análisis fueron el origen de una renovación profunda de nuestras concepciones que iba mucho más allá de su propio dominio de estudio. La originalidad primera de su enfoque, cada uno lo sabe, fue no partir de una definición previa de la clase obrera sino seguir las etapas y las formas de un proceso - the making - que, entre las últimas décadas del siglo XviII y las primeras del xIX, condujo a la constitución y a la afirmación de una nueva entidad social en la que la presencia se volvió evidente a comienzos de los años i83o. El historiador define esta clase "como un fenómeno histórico, uniendo los eventos sueltos y sin vínculos aparentes, tanto en la objetividad de la experiencia cuanto en la consciencia [de los actores sociales]» (THOMPSON, I988: 38 ).

Thompson no es ciertamente un microhistoriador, más bien todo lo contrario. Aborda el estudio de un fenómeno masivo en toda su amplitud. En todo su espesor también, y sobre este punto, plantea preguntas que nos interesan directamente. Porque estos «eventos dispares y sin vínculos aparentes» que le permiten captar lo que «sucedió en las relaciones humanas» se sitúan en otro nivel que el del

4] La primera versión de esta obra de E.P. THOMPSON fue editada en 1963. 
fenómeno global que es el punto de llegada y la resultante de una multiplicidad de transformaciones más limitadas, más locales. Porque esta es la tesis central del libro: hubo, en Inglaterra, muchas formas, «dispares», a veces contradictorias, de participar en la constitución de la clase obrera; hubo también diversos momentos en este proceso, momentos y formas que es importante seguir sin presuponer el carácter ineludible del punto de llegada. Sean formas de la agregación social o de su dinámica, las transformaciones se inscriben sobre unos planos diferentes, que el historiador debe imponerse respetar.

Todavía una vez más importa no hacer decir a Thompson lo que no dijo, y puedo imaginar que él habría sido particularmente reticente al tema de la discontinuidad sobre la cual puse el acento aquí. En su perspectiva analítica, sin embargo, creo que tomar en cuenta el desplazamiento, a veces heterogéneo entre varios mundos y diversas modalidades de la experiencia social, es esencial para su demostración. El macroproceso del que él da cuenta, la formación de la clase obrera, no se comprende que a condición de captarlo en sus diferentes escalas a las cuales le corresponden lógicas sociales que son también diferentes.

Es en este punto que me parece se podría con justeza situar en la vía abierta por Thompson un cierto número de trabajos que se reivindicaron como microhistoria. Fue muy evidente el caso de Edoardo Grendi, uno de los historiadores que más se dedicó a dar a conocer la obra del historiador británico en Italia y en el que se sabe el rol que él jugó en la elaboración de la propuesta de microhistórica ${ }^{5}$. De la generación posterior, se puede, por ejemplo, pensar en la búsqueda de Maurizio Gribaudi sobre las formas que tomó la integración de inmigrantes venidos del mundo rural de la ciudad industrial - Torino y la Fiat en el caso estudiadodurante las primeras décadas del siglo xx. Aun si se propuso seguir trayectorias individuales, su proyecto siguió siendo el de «investigar los diferentes materiales a partir de los cuales se habían construido las diversas experiencias y fisionomías obreras y de este modo iluminar, así, las dinámicas que habían permitido la agregación como las disgregaciones» (GRIBAUDI, I987: 27).

De tal modo de proceder, otro ejemplo, más radical todavía, me permitirá quizá ilustrar más eficazmente mi propósito. Entre mediados del siglo XIX y las

5] Cabe recordar que Grendi fue quien impulsó la traducción y prologó una extensa compilación de los principales artículos de E.P. Thompson, que se editó en 1981 en italiano: Società patrizia e cultura plebea, Turín, Einaudi. 
primeras décadas del xx, Francia conoció, con relativo retraso, una transferencia masiva de la población rural hacia las ciudades, en particular hacia Paris. Al tema del «éxodo rural»se habían vinculados numerosas consideraciones ideológicas que dicho término evocaba: la amenaza de una desertificación de los campos, la invasión que amenazaba las ciudades, la contaminación de los espacios sociales y las desgracias que debían acompañarla, el miedo de una ruptura de equilibrio entre «París y el desierto francés», para retomar una fórmula que se volvería ritual. Este fenómeno fue bien conocido. La amplitud y la cronología de la transferencia podían ser detalladas a través de los censos de la población. En la mayoría de los casos se interpretó como un fenómeno inevitable, ejercido al mismo tiempo por los desequilibrios del empleo, el diferencial de salarios, por la atracción de un modo de vida individualista urbano, en favor de la movilidad vuelta posible por el ferrocarril, las dinámicas determinadas por los grandes ciclos económicos, etc. No hay nada más para decir de esta interpretación sino que ella fue construida a partir de los resultados globales de un desplazamiento sociodemográfico —es decir a partir de su punto de llegada - de ahí el carácter de inevitabilidad que le han conferido la mayor parte de los análisis existentes. Paul-André Rosental eligió tomar las cosas desde otro lado, invirtiendo la perspectiva: es decir estudiar las realidades migratorias a partir de su punto de partida, siguiendo a Gribaudi y antes de él a Thompson (ROSENTAL, I999).

Ello implicó reconstruir las trayectorias nominativas para intentar comprender, lo más cerca posible lo que significó la experiencia de los actores, los motivos, las representaciones del espacio social, las formas de solidaridad — familiar, de grupos etarios, profesional - y de inscripción que estuvieron en el origen de la elección de los desplazamientos efectuados. Le fue de esta manera posible reconstruir los «senderos invisibles» de la migración; aquellas vías que fueron ocultadas por los datos masivos, más atentos a describir e interpretar el proceso del «éxodo rural»y menos a recuperar la discontinuidad y el carácter no lineal de dichos caminos de migración. Más allá de las intenciones de los actores, que no son jamás explícitos, Rosental puso en valor los comportamientos diferenciados de configuraciones interpersonales locales que habían favorecido en diverso modo la movilidad geográfica y profesional en el seno del linaje familiar, diferenciando los patrones de aquellas autocentradas respecto de las exocentradas. Comúnmente se considera, observa Rosental, «que los migrantes reaccionan inmediata y mecánicamente a los estímulos, los cuales son exteriores e inscriptos en un contexto macroscópico» 
(ROSENTAL, 1999: 36). La opción de situar su indagación en una escala microanalítica no solo ilumina otra clase de hechos, sino que permite aprehender lógicas sociales de diferente naturaleza, que no son legibles e interpretables más que a ese nivel, y que transforman considerablemente lo que nosotros podemos saber del fenómeno global de la migración. De este modo llega a la conclusión que «los habitantes rurales no respondieron espontáneamente a vastas transformaciones macroeconómicas, que habrían vuelto insoportable su permanencia en el campo. Es porque aquellas pesaron en las dinámicas internas de las familias y en las características propias de cada linaje, que han, según el caso, fomentado la movilidad o el sedentarismo" (ROSENTAL, I999: 198).

Aun cuando el fenómeno nos parece acumulativo en sus efectos agregados en la larga duración, en la pequeña escala puede ser considerado como un proceso laminado, que obedece a lógicas discontinuas, que según el nivel de análisis en el cual uno se sitúa pueden aparecer como dinámicas superpuestas y otras divergentes. Todo fenómeno social mayor, es sin dudas repensable desde este tipo de análisis, sean realidades socioeconómicas, transformaciones culturales - pensemos en los mecanismos de alfabetización- ${ }^{6}$ o de un partido político.

IV

Empero los fenómenos colectivos masivos no son los únicos que requieren de este tipo de experiencia. De allí que, de manera repetida, la biografía se haya encontrado en el centro del debate historiográfico durante los últimos tres decenios. Se trata, se sabe, de un género sobre todo tradicional, que los historiadores profesionales practican de buen grado, aunque con sospecha y condescendencia, como si su legitimidad presentara visos de incerteza. La vitalidad y éxito del género reposan sobre su amplia recepción en un público muy diverso, matrizado por las expectativas e inclinaciones del consumismo cultural que van más allá del círculo de profesionales. Este hecho ha contribuido, sin dudar, a la prevención de la que es objeto. La biografía borra las fronteras acostumbradas, las cabalga sin grandes atenciones, lo hace tanto más fácilmente en cuanto que puede ser declinada según

6] Un excelente ejemplo de esta perspectiva metodológica aplicada a transformaciones socioculturales en la compilación de FURET Y OZOUF (1976). 
formas muy diversas. La producción es masivamente tradicional. Esto no impide que este género historiográfico haya puesto en escena cuestiones importantes y que, en mi opinión, van más allá del género biográfico mismo. La crítica devastadora de la «ilusión biográfica» que formuló Pierre Bourdieu (BOURDIEU, 1986) puede así ser extendida a toda una gama de realidades sociales cuya existencia los historiadores, por costumbre o por pereza, voluntariamente han considerado como un dato. Ella nos invita a interrogarnos sobre las categorías de análisis, las modalidades, las técnicas que nos permiten construir y validar en un texto la representación de una vida. Continuando la crítica inicial de Bourdieu, Giovanni Levi ha demostrado provechosamente lo que es posible extraer de una reflexión sobre los usos de la biografía (LEVI, I989). Usualmente, tenemos tendencia a pensar que una vida - comenzando por la propia - es un conjunto coherente y continuo, privilegiando en el análisis lo que refuerza la coherencia y el encadenamiento de secuencias. Solo las experiencias límites desafían y cuestionan radicalmente este relato lineal. La obra de Laurence Sterne, escrita alrededor de 1769, Tristam Shandy es con justicia el ejemplo clásico. Desde entonces, con Dostoïevski, Proust, Kafka o Musil, la novela se concedió formas de experimentación que volvieron familiar el cuestionar el estatuto del personaje. Es verdad que el novelista dispone de una libertad que nos está prohibida. Un historiador tiene sin embargo, el derecho de realizar las preguntas más complejas: ¡cómo fijar los contornos, es decir, los límites de una biografía? ¿Cómo discriminar lo que es pertinente de lo que no lo es en este tema?7.

Detengámonos un instante sobre una experimentación llevada a cabo hace más de medio siglo por el medievalista italiano Arsenio Frugoni (Frugoni, 1954). Arnaldo da Brescia, a quien él le consagró un estudio memorable, era un reformador religioso de la primera mitad del siglo XII, que es conocido gracias a un número limitado de fuentes de origen y naturaleza muy diversas, las cuales, además, se contradicen drásticamente. El conjunto suma diez colecciones documentales que van desde las crónicas de la ciudad de Brescia a las cartas de Saint Bernard, de Otto de Freising —el gran hombre de la diplomacia imperial—, al humanista Jean de Salisbury e incluso llegan hasta un anónimo poeta lombardo. En estos textos, no son solamente los juicios puestos en la persona y en el accionar de Arnaldo los que difieren profundamente, sino fundamentalmente lo que se nos cuenta de los

7] Precisamente es la pregunta que se hace Giovanni Levi en la introducción a su libro L'Eredità immateriale. Carriera di un esorcista nel Piemonte del Seicento (LEVI, 1985: 9). 
acontecimientos de su vida. De un historiador riguroso, se espera que se esfuerce en cotejar estos testimonios y que no conserve más que la información que es posible verificar. Sin embargo, esta no fue la elección de Frugoni. Sino que, como el mismo lo explica, rechazó ceder al «método de la combinación», para retomar cada fuente en sus propios términos, lo que conllevaría a integrar el máximo de información siempre conservando únicamente los datos que pueden inscribirse en un retrato verosímil — ¿verosímil para quién?- del personaje, «como si se tratara de piezas perfectas de un mosaico»; como si, escribe Frugoni, «la Providencia [fuera] siempre tan benévola con los historiadores que les ofrece[ría] todos los elementos que permiten una reconstrucción biográfica satisfactoria» (FRUGONI, I954: I2). Para mejor resistir a esta tentación, el historiador eligió, entonces, consagrar diez capítulos de su libro a una fuente particular. De esto resulta un retrato fragmentado, y naturalmente, contradictorio; diez versiones de Arnaldo, que no hay que necesariamente comprender como la confesión de un relativismo escéptico del tipo «a cada uno su verdad». Lo que se busca, al contrario, es la verdad de cada fuente en el contexto específico — político, religioso, cultural, relacional— en el que ella encuentra su significación. Al optar por la discontinuidad nos ofrece un retrato y una lectura del reformador mucho más compleja puesto que da cuenta, tanto como es posible, de estas contradicciones, de estos fragmentos de información voluntariamente tratados como segmentos. Esta estrategia metodológica hace posible la reconstrucción de diferentes planos de la experiencia biográfica de Arnaldo, los diversos mundos de los que formó parte en su vida — hasta donde dejan percibir los documentos - sin prejuzgar la integración posible de estos mundos en una experiencia única que no sabría ser más que una reconstrucción posterior no susceptible, por tanto, de verificación.

Así, se podrían multiplicar los ejemplos de fenómenos que comúnmente son pensados como globales u holísticos y que podrían beneficiarse con aproximaciones en diferentes escalas, para ser aprehendidos con una mayor complejidad. Para no evocar más que uno, importante sin duda, hay un cierto número de trabajos recientes que intentaron volver a preguntarse sobre la historia del Estado moderno en los distintos niveles de su construcción concreta. A menudo se piensa el Estado como una gran arquitectura centralizada y donde las formas no pararían de multiplicarse, de ramificarse, hasta penetrar, en lo más profundo de la sociedad que aspira a controlar — cuadro que, por otra parte, replica la imagen que el discurso estatal busca dar de sí mismo-. En estos nuevos estudios, en cambio, el Estado 
moderno aparece como una realidad un poco más complicada, menos armoniosa, menos imperiosa también. En lugar de construir explicaciones a partir de la cuantificación y la construcción de índices globales — del número de sus agentes, la carga fiscal, la densidad de sus instituciones y de sus normas-, se busca hace valer la importancia de los ajustes locales, de las transacciones necesarias con el mundo social, de las discontinuidades institucionales. Evidentemente, esto no conduce a afirmar que el Estado no existe en esos niveles, lo que no tendría mucho sentido. Sino que él no existe necesariamente bajo las formas en las que se lo reconoce - y se reconoce a sí mismo- cómodamente, y que solo existe a condición de convertirse en otra cosa. Observemos que en este punto, la oposición entre macro y micro resulta insuficiente ya que es deseable multiplicar los niveles intermedios que, en el caso del Estado en particular, permiten tomar la plena complejidad del fenómeno. Toda realidad histórica mayor toma sin duda forma y sentido, como es el caso del Estado, en una pluralidad de mundos sociales.

De una variación de las escalas de observación, se puede entonces esperar un beneficio analítico en el enfoque de los fenómenos sociales. De una puesta en valor de las discontinuidades detectables entre los diferentes niveles, se puede esperar un enriquecimiento de las hipótesis y de las interpretaciones que somos capaces de formular.

\section{V}

A estas razones, deseo agregar una última. Porque nosotros, los historiadores, venimos après coup normalmente tenemos tendencia a racionalizar la existencia, lo que es también una manera de legitimarla: las cosas acontecieron como acontecieron. Es el problema de la «retrodicción», esa forma de percepción ya comentada por Hannah Arendt, que está ligada a la posición del historiador en relación a su objeto. Es desde esta postura cognitiva coaccionada que nosotros sacamos, muy seguido, unas conclusiones sobre el carácter ineludible de los procesos sociales, se trate de la construcción del Estado moderno, del éxodo rural, de la industrialización y de la urbanización, y por supuesto, bajo nuestros ojos, de la «globalización» en curso. En todos estos casos, y en muchos otros también, el asir un fenómeno a través de los datos agregados, contribuye a reforzar la coherencia y el carácter necesario. Una vez más, no se trata de poner en duda la realidad de los procesos que operan 
para unir las sociedades humanas, en particular hoy. Sino de mostrar que ellos se basan, en un desfasaje entre los niveles donde se puede captar los efectos y las respuestas que generan. ¿Quién no ve que un fenómeno contemporáneo como la globalización, que no cesa de recordarnos que produce unos efectos inevitables, provoca a su vez, unas formas nuevas de diferenciación? En este sentido, puede ser significativo que, en un momento donde se considera tan fuertemente adquirida la unificación de las sociedades humanas, un cierto número de historiadores (pero también de sociólogos y de antropólogos) se hayan vuelto más atentos a los fenómenos de discontinuidad. Ellos encuentran aquí, sin duda, un instrumento crítico frente a las evidencias simplificadoras de la realidad del mundo histórico. Y quizá también, como lo anhelaba Eduardo Hourcade, encuentran unas razones renovadas para practicar el antiguo oficio.

\section{Referencias bibliográficas}

BOURDIEU, PIERRE (1986): «L'illusion biographique», GONZÁLEZ Y GONZÁLEZ, LUIS (1982): Nueva invitación en: Actes de la recherche en sciences sociales, a la microhistoria, Secretaría de Educación Pú$n^{0}$ 62/63, pp. 69-72.

BRAUDEL, FERNAND (1949): La Méditerranée et le Monde méditerranéen à l'époque de Philippe II, II t., París, Armand Collin. blica/Fondo de Cultura Económica. GRIBAUDI, MAURIZIO (1987): Itinéraires ouvriers. Espaces et groupes sociaux à Turin au début du XXe siècle, París, Éditions de l'EHESS.

BRAUDEL, FERNAND (1972): Ecrits sur l'histoire, Paris, Flammarion.

LEVI, GIOVANNI (1985): L'Eredità immateriale. CaFRUGONI, ARSENIO (1954): Arnaldo da Brescia nelle fonte de/ secolo XII, Rome, Istituto Storico Italiano per il Medioevo.

FURET, FRANÇOIS Y OZOUF, MONA (1976): Lire et écrire. L'alphabétisation des Français de Luther à Jules Ferry, t. 1, París, Minuit.

GONZÁLEZ Y GONZÁLEZ, LUIS (1973): Invitación a la microhistoria, México, Secretaría de Educación Pública/Fondo de Cultura Económica.

GONZÁLEZY GONZÁLEZ, LUIS ([1968] 1977): Les barrièrriera di un esorcista nel Piemonte del Seicento, Turín, Einaudi.

LEVI, GIOVANNI (1989): "Les usages de la biographie», en : Annales ESC, nº 6, pp. 1325-1336. ROSENTAL, PAUL-ANDRÉ (1999): Les sentiers invisibles. Espace, familles et migrations dans la France du $19^{\circ}$ siècle, París, Éditions de l'EHESS. THOMPSON, EDWARD P. (1981): Società patrizia e cultura plebea, Turín, Einaudi.

THOMPSON, EDWARD P. (1988): La formation de la classe ouvrière anglaise, París, Gallimard/Seuil.

res de la solitude. Histoire universelle de San José de Gracia, village mexicain, París, Plon. 